\title{
Prohibitin 1 Modulates Mitochondrial Stress-Related Autophagy in Human Colonic Epithelial Cells
}

\author{
Arwa S. Kathiria ${ }^{1}$, Lindsay D. Butcher ${ }^{1,2}$, Linda A. Feagins ${ }^{3}$, Rhonda F. Souza ${ }^{3}$, C. Richard Boland ${ }^{1}$, \\ Arianne L. Theiss ${ }^{1 *}$
}

1 Division of Gastroenterology, Department of Internal Medicine, Baylor Research Institute, Baylor University Medical Center, Dallas, Texas, United States of America, 2 Institute of Biomedical Studies, Baylor University, Waco, Texas, United States of America, 3 Department of Medicine, Veterans Affairs North Texas Health Care System, University of Texas Southwestern Medical Center, Dallas, Texas, United States of America

\begin{abstract}
Introduction: Autophagy is an adaptive response to extracellular and intracellular stress by which cytoplasmic components and organelles, including damaged mitochondria, are degraded to promote cell survival and restore cell homeostasis. Certain genes involved in autophagy confer susceptibility to Crohn's disease. Reactive oxygen species and pro-inflammatory cytokines such as tumor necrosis factor $\alpha(T N F \alpha)$, both of which are increased during active inflammatory bowel disease, promote cellular injury and autophagy via mitochondrial damage. Prohibitin (PHB), which plays a role in maintaining normal mitochondrial respiratory function, is decreased during active inflammatory bowel disease. Restoration of colonic epithelial PHB expression protects mice from experimental colitis and combats oxidative stress. In this study, we investigated the potential role of PHB in modulating mitochondrial stress-related autophagy in intestinal epithelial cells.
\end{abstract}

Methods: We measured autophagy activation in response to knockdown of PHB expression by RNA interference in Caco2BBE and HCT116 WT and p53 null cells. The effect of exogenous PHB expression on TNF $\alpha$ - and IFN $\gamma$-induced autophagy was assessed. Autophagy was inhibited using Bafilomycin $\mathrm{A}_{1}$ or siATG16L1 during PHB knockdown and the affect on intracellular oxidative stress, mitochondrial membrane potential, and cell viability were determined. The requirement of intracellular ROS in siPHB-induced autophagy was assessed using the ROS scavenger $N$-acetyl-L-cysteine.

Results: TNF $\alpha$ and IFN $\gamma$-induced autophagy inversely correlated with PHB protein expression. Exogenous PHB expression reduced basal autophagy and TNF $\alpha$-induced autophagy. Gene silencing of PHB in epithelial cells induces mitochondrial autophagy via increased intracellular ROS. Inhibition of autophagy during PHB knockdown exacerbates mitochondrial depolarization and reduces cell viability.

Conclusions: Decreased PHB levels coupled with dysfunctional autophagy renders intestinal epithelial cells susceptible to mitochondrial damage and cytotoxicity. Repletion of PHB may represent a therapeutic approach to combat oxidant and cytokine-induced mitochondrial damage in diseases such as inflammatory bowel disease.

Citation: Kathiria AS, Butcher LD, Feagins LA, Souza RF, Boland CR, et al. (2012) Prohibitin 1 Modulates Mitochondrial Stress-Related Autophagy in Human Colonic Epithelial Cells. PLoS ONE 7(2): e31231. doi:10.1371/journal.pone.0031231

Editor: Giovambattista Pani, Catholic University Medical School, Italy

Received October 12, 2011; Accepted January 4, 2012; Published February 17, 2012

Copyright: (C) 2012 Kathiria et al. This is an open-access article distributed under the terms of the Creative Commons Attribution License, which permits unrestricted use, distribution, and reproduction in any medium, provided the original author and source are credited.

Funding: This work was supported by National Institutes of Health grant K01-DK085222 (A.L.T.) and funds from the Baylor Research Institute. The funders had no role in study design, data collection and analysis, decision to publish, or preparation of the manuscript.

Competing Interests: The authors have declared that no competing interests exist.

* E-mail: arianne.theiss@baylorhealth.edu

\section{Introduction}

Autophagy is an evolutionarily conserved catabolic pathway that degrades cytoplasmic components such as long-lived proteins, macromolecules and damaged organelles including the endoplasmic reticulum and mitochondria through lysosomal degradation $[1,2,3]$. Autophagy is an adaptive response to extracellular stress, such as starvation, or intracellular stress, including the accumulation of misfolded proteins, damaged organelles, or the invasion of microorganisms, intended to promote cell survival and restore cell homeostasis [4]. Either apoptosis or autophagic cell death can be initiated in irreversibly damaged cells [5]. Malfunctioning autophagy has been associated with multiple diseases such as cancer, neurodegeneration, autoimmune diseases and inflammatory diseases, including inflammatory bowel disease (IBD) [6].
The two common, but disparate, forms of IBD, Crohn's disease and ulcerative colitis, share related characteristics such as mucosal damage and diarrhea but have distinguishing clinical features. The etiopathogenesis of IBD remains unknown but is thought to involve a combination of genetic and non-genetic risk factors that regulate mucosal immune response, mucosal barrier function, and response to microbial factors [7]. Multiple epithelial molecules have been identified as mediators of IBD pathogenesis including those that control epithelial homeostasis [8]. Genome-wide association studies and meta-analysis have identified the autophagy genes ATG16L1, IRGM, and LRRK2 as candidate loci involved in genetic susceptibility to Crohn's disease $[9,10,11,12]$. Mutation or deletion of ATG16Ll results in increased proinflammatory cytokine production, increased susceptibility to experimental colitis, and reduced capability to eradicate invading 
bacteria, indicating the importance of autophagy in suppressing intestinal inflammation [13,14,15].

Multiple studies have reported mitochondrial dysfunction in Crohn's disease and ulcerative colitis $[16,17,18,19]$ as well as the dextran sodium sulfate and 2,4,6-trinitrobenzene sulfonic acid models of colitis [20,21]. Mitochondria are important regulators of autophagy and apoptosis. During normal function of the mitochondrial respiratory chain, reactive oxygen species (ROS), which are partially reduced oxygen species such as superoxide radical $\left(\mathrm{O}_{2}^{-}\right)$, hydrogen peroxide $\left(\mathrm{H}_{2} \mathrm{O}_{2}\right)$, hydroxyl radical $(\cdot \mathrm{OH})$, and peroxynitrate $\left(\mathrm{NOO}^{-}\right)$, are generated at low levels. Production of ROS is increased when mitochondria are damaged [22]. IBD is associated with increased ROS and decreased antioxidant enzymes in the intestinal mucosa $[23,24,25,26]$. It is widely accepted that ROS produced as a by-product of respiration as well as exogenous ROS can induce autophagy via mitochondrial damage $[27,28]$. Mitochondria are the main source of ROS for regulation of autophagy [29]. In fact, exogenous ROS and the proinflammatory cytokine tumor necrosis factor $\alpha(\mathrm{TNF} \alpha)$, both of which are increased during IBD, promote cellular injury and autophagy via mitochondrial ROS generation [29,30,31]. Defects in autophagy result in the accumulation of intracellular ROS and deformed mitochondria [14,32].

Prohibitin 1 (PHB) is an evolutionarily conserved, multifunctional $32 \mathrm{kDa}$ protein implicated in cellular processes including the regulation of proliferation, apoptosis, and transcription $[33,34,35,36]$. PHB is predominantly localized to the mitochondria in intestinal epithelial cells [37] and multiple studies have shown that PHB plays a role in maintaining normal mitochondrial function and morphology (reviewed in [38]). It has been shown that PHB interacts with complex I and subunits of cytochrome $c$ oxidase of the respiratory chain and regulates their assembly $[39,40]$. Loss of PHB in mitochondria impairs function of the mitochondrial respiratory chain $[39,40]$. One obvious effect of respiratory chain dysfunction is increased oxidant production leading to oxidative stress, which can cause alterations in mitochondrial morphology and membrane potential [29].

Expression of PHB is decreased in mucosal biopsies from ulcerative colitis and Crohn's disease afflicted patients and in animal models of colitis $[37,41]$. Pro-inflammatory cytokines such as $\mathrm{TNF} \alpha$ and oxidative stress induced by exogenous $\mathrm{H}_{2} \mathrm{O}_{2}$ decrease expression of intestinal epithelial PHB in vivo and in vitro $[37,42]$. Restoration of colonic epithelial PHB expression using genetic manipulation (villin-PHB transgenic mice) or therapeutic delivery to the colon via nanoparticle or adenovirus protected mice from experimental colitis [43,44]. Our recent data suggest that epithelial PHB sustains anti-oxidant expression [44] and has anti-inflammatory properties such as reducing $\mathrm{TNF} \alpha$-stimulated $\mathrm{NF}-\kappa \mathrm{B}$ activation [42]. This is in agreement with emerging data that suggest a role of $\mathrm{PHB}$ in combating oxidative stress in multiple cells types $[39,40,45,46]$. In this study, we investigated the potential role of PHB in modulating mitochondrial stress-related autophagy in intestinal epithelial cells. Here, we show that TNF $\alpha$ and IFN $\gamma$-induced autophagy inversely correlates with PHB protein expression and that gene silencing of $\mathrm{PHB}$ induces mitochondrial autophagy via increased intracellular ROS. Inhibition of autophagy during PHB knockdown exacerbates mitochondrial depolarization and reduces cell viability. These data suggest that decreased PHB levels coupled with dysfunctional autophagy renders intestinal epithelial cells susceptible to mitochondrial ROS and cytotoxicity.

\section{Results}

PHB protein expression inversely correlates with cytokine-induced autophagy in cultured colonic epithelial cells

Our previous studies showed that $\mathrm{TNF} \alpha$ reduces expression of PHB in intestinal epithelial cells in vivo and in vitro [42]. Proinflammatory cytokines such as TNF $\alpha$ and IFN $\gamma$ have been shown to induce autophagy in human intestinal epithelial cell lines [31,47]. Confluent monolayers of Caco2-BBE cells were treated with $10 \mathrm{ng} / \mathrm{ml} \mathrm{TNF} \alpha$ or $50 \mathrm{ng} / \mathrm{ml} \mathrm{IFN} \gamma$ alone or in combination for 18 hours. As expected, $\mathrm{TNF} \alpha$ and IFN $\gamma$ increased two biochemical signs of autophagy: the conversion of LC3-I to LC3-II, indicated by normalizing LC3-II to LC3-I protein levels, and increased beclin-1 protein expression (Figure $1 \mathrm{~A}$ and $1 \mathrm{~B}$ ) $[27,48]$. Conversely, PHB protein levels in the same samples were decreased by TNF $\alpha$ and IFN $\gamma$ (Figure 1A and 1B). The effect of $\mathrm{TNF} \alpha$ and IFN $\gamma$ given in combination reflected that of cells treated with either cytokine alone and therefore, we did not pursue the effects of these cytokines in combination. It is widely accepted that the tumor suppressor p53 regulates autophagy [49]. Since Caco2-BBE cells have mutated p53 [50], we assessed the involvement of p53 in autophagy induction by $\mathrm{TNF} \alpha$ and IFN $\gamma$ in wild-type (WT) and p53 null HCT116 colonic epithelial cells. HCT116 cells, including p53 null cells [51], also showed the conversion of LC3-I to LC3-II and increased beclin-1 protein expression suggesting that the effect of $\mathrm{TNF} \alpha$ and $\mathrm{IFN} \gamma$ to increase autophagy and decrease $\mathrm{PHB}$ protein expression is independent of p53 signaling (Figure 1C and 1D).

\section{Exogenous PHB expression reduces basal autophagy and TNF $\alpha$-induced autophagy in intestinal epithelial cells}

Since PHB expression inversely correlated with the induction of autophagy in colonic epithelial cells, we determined whether exogenous PHB expression could affect autophagy. Caco2-BBE cells stably overexpressing GFP-tagged PHB (pEGFPN1-PHB) show decreased LC3-II conversion from LC3-I and reduced beclin-1 protein expression (Figure 2A and 2B), suggesting a reduction in basal autophagy. Treatment with $\mathrm{TNF} \alpha$ or IFN $\gamma$ increased LC3-II and beclin-1 protein abundance in empty vector expressing cells (Figure 2A and 2B), reflecting the same response as WT Caco2-BBEs in Figure 1A. PHB overexpressing cells showed decreased TNF $\alpha$-induced LC3-I conversion to LC3-II and beclin1 protein expression compared to vector-transfected cells, whereas PHB overexpression did not affect IFN $\gamma$-induced LC3-II or beclin-1 protein expression (Figure 2A and 2B). Since PHB overexpression did not decrease IFN $\gamma$-induced autophagy, this would suggest that the IFN $\gamma$ autophagy pathway is distinct from that of TNF $\alpha$. An antibody specific to GFP was used to assess protein expression of GFP-PHB and GFP in PHB and vector overexpressing cells, respectively.

\section{Knockdown of PHB induces autophagy}

Since PHB protein expression inversely correlated with $\mathrm{TNF} \alpha$ and IFN $\gamma$-induced autophagy in Caco2-BBE cells, we next determined whether knockdown of PHB expression could induce autophagy. Caco2-BBE cells transfected with siPHB showed conversion of LC3-I into LC3-II (Figure 3A) as well as increased beclin-1 protein expression (Figure 3B) compared to cells transfected with a negative control siRNA (siNeg ctl). PHB knockdown stimulated the redristribution of GFP-LC3 fusion protein from a diffuse signal to cytoplasmic puncta indicative of autophagosomes (Figure 3G). The mitochondrial stress proteins 
A Caco2-BBE

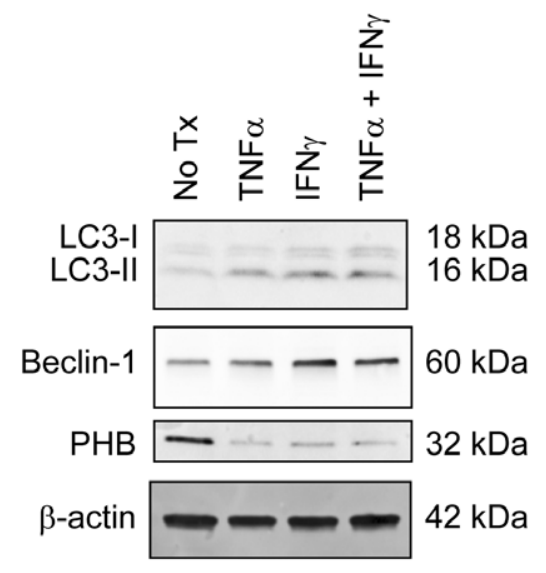

HCT116

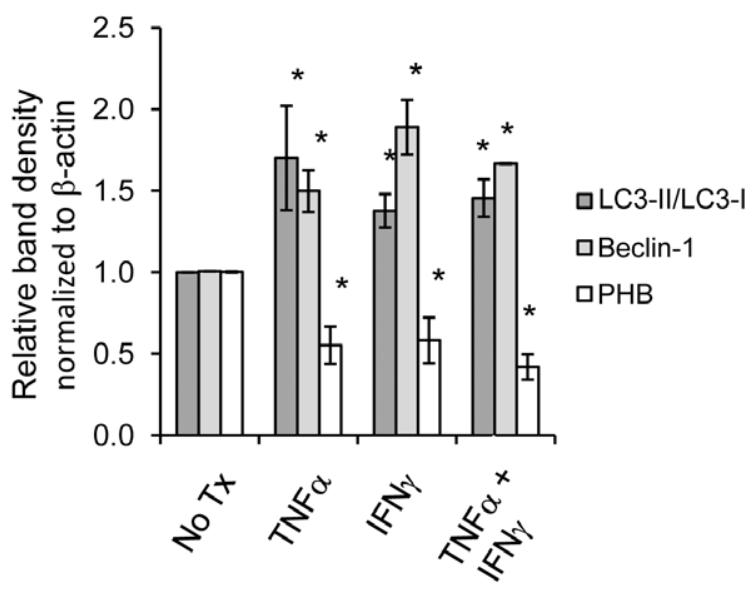

$\mathrm{D}$
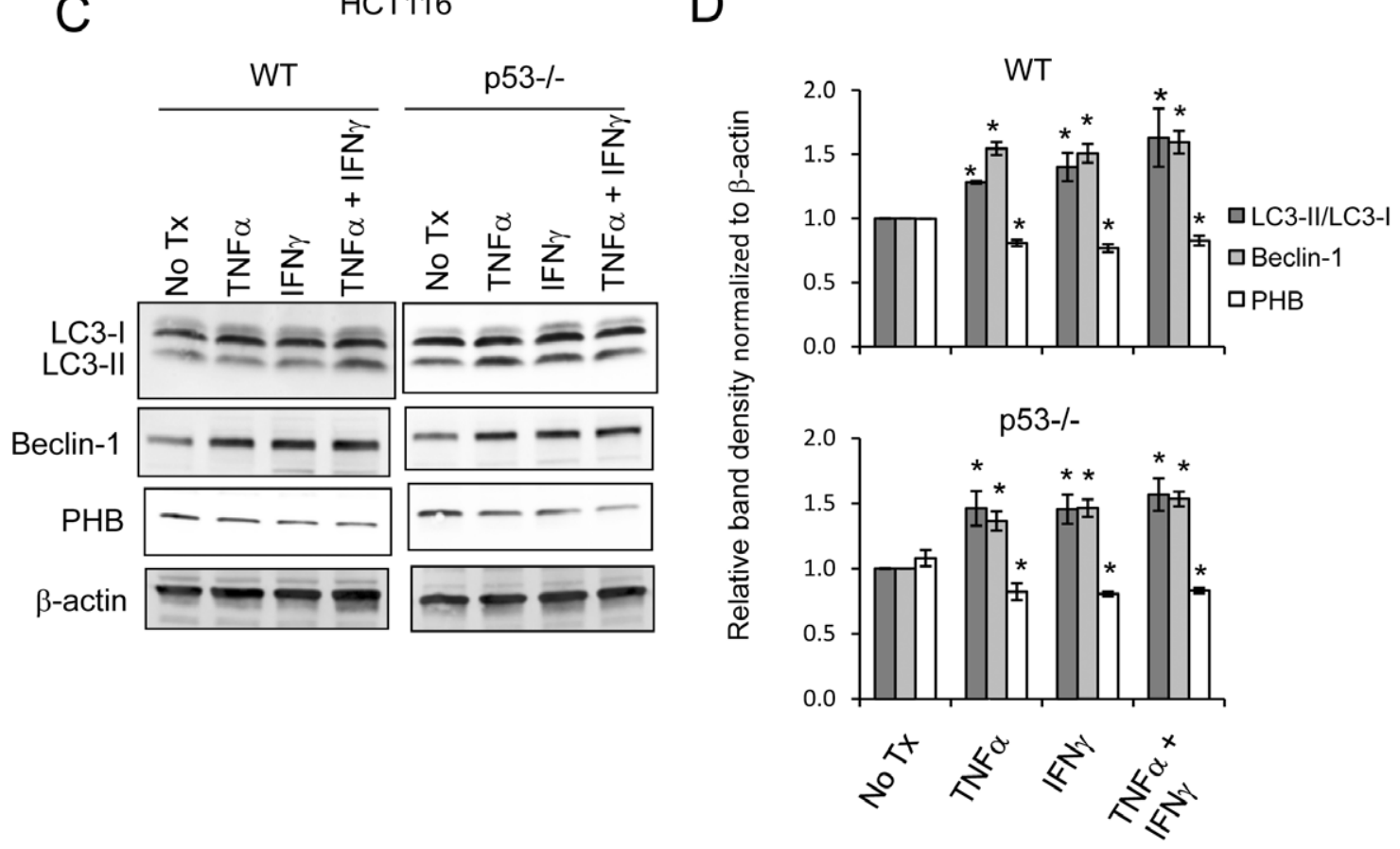

Figure 1. PHB protein levels inversely correlate with cytokine-induced autophagy in cultured colonic epithelial cells. (A) Representative Western blots showing LC3I/LC3II, Beclin-1, PHB or $\beta$-actin (loading control) expression in Caco2-BBE cells treated with $10 \mathrm{ng} / \mathrm{ml}$ TNF $\alpha$ or $50 \mathrm{ng} / \mathrm{ml} \mathrm{IFN} \gamma$ alone or in combination for 18 hours. (B) Histograms show mean \pm SEM relative to no treatment control Caco2-BBE cells. ${ }^{*} P<0.05$ vs. no tx; $n=3$. (C) Representative Western blots showing LC3I/LC3II, Beclin-1, PHB or $\beta$-actin (loading control) expression in HCT116 cells treated with $10 \mathrm{ng} / \mathrm{ml} \mathrm{TNF} \alpha$ or $50 \mathrm{ng} / \mathrm{ml} \mathrm{IFN} \gamma$ alone or in combination for 18 hours. (D) Histograms show mean \pm SEM relative to no treatment control HCT116 cells. ${ }^{*} P<0.05$ vs. no $\mathrm{tx} ; \mathrm{n}=3$.

doi:10.1371/journal.pone.0031231.g001

Cpn60, PKR, and ClpP showed no change in expression by Western blot upon PHB knockdown suggesting that inhibition of PHB does not induce the mitochondrial unfolded protein response (Figure S1). The efficiency of siRNA knockdown was validated by western blot (Figure 3A). Protein expression of PHB was reduced $\sim 80 \% 96$ hours after transfection.

The effect of PHB knockdown in Caco2-BBE cells was corroborated in HCT116 cells. Knockdown of PHB induced the conversion of LC3-I to LC3-II, increased beclin-1 protein expression (Figure $4 \mathrm{~A}$ and $4 \mathrm{~B}$ ) and increased the formation of cytoplasmic puncta by the GFP-LC3 fusion protein (Figure 4C). Although knockdown of PHB in HCT116 cells (approximately $50 \%$ reduction compared to control; Figure $4 \mathrm{~A}$ ) was not as strong as in Caco2-BBE cells, the results on autophagy were similar to that in Caco2-BBE cells. p53 inhibition, including p53 knockout by homologous recombination, initially induces autophagy by ER stress followed by mitophagy (autophagy of mitochrondria) [52]. To determine whether the induction of autophagy by PHB 
Caco2-BBE
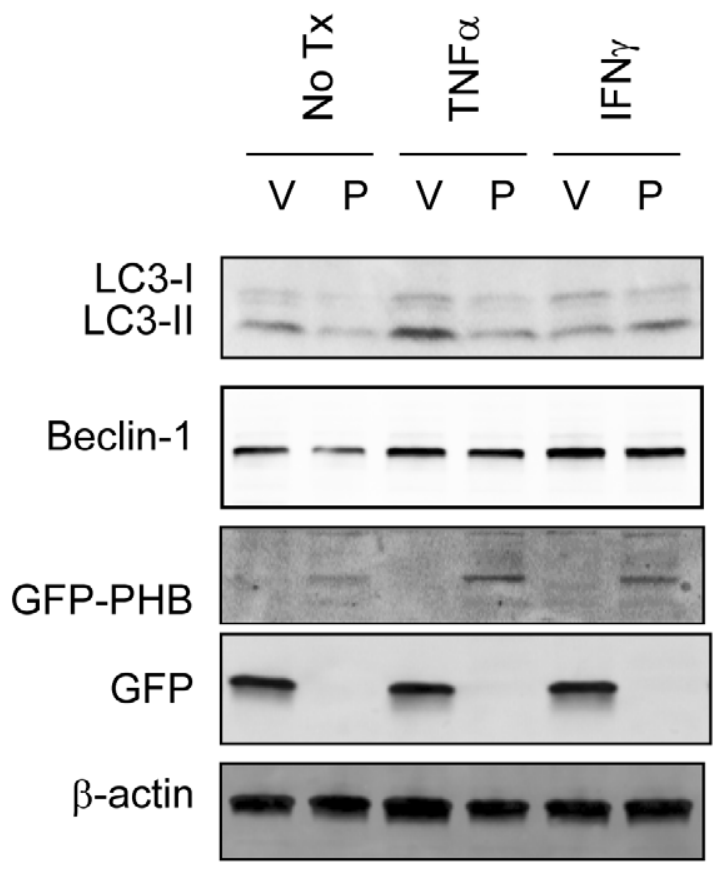

B
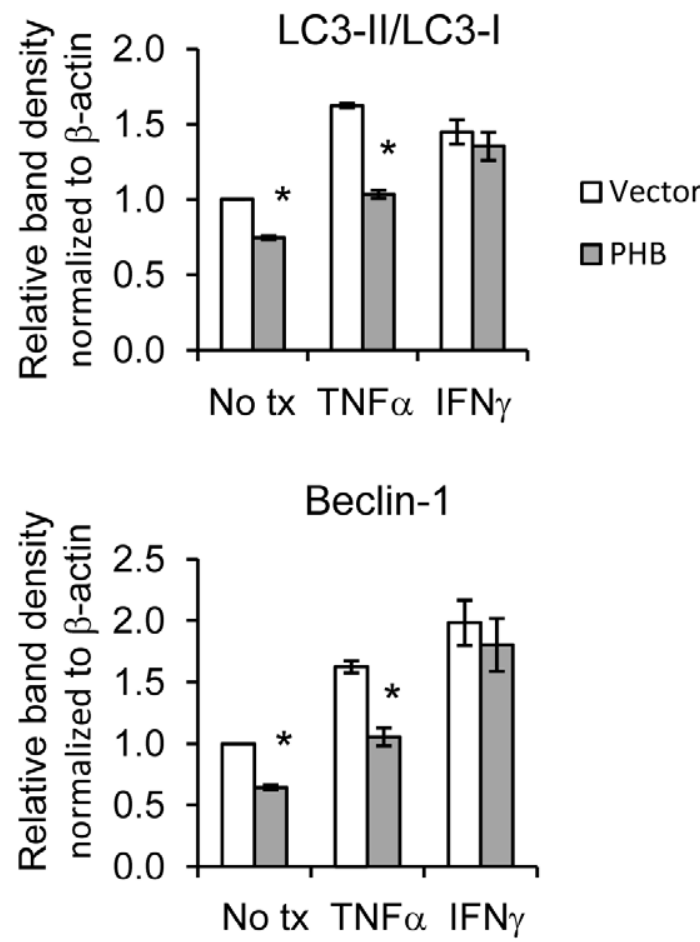

Figure 2. Exogenous PHB expression reduces basal autophagy and TNFo-induced autophagy in intestinal epithelial cells. (A) Representative Western blots of 3 independent experiments showing LC3I/LC3II, Beclin-1, GFP-PHB, GFP or $\beta$-actin (loading control) expression in Caco2-BBE cells transfected with either pEGFPN1 expression vector $(V)$ or pEGFPN1-PHB (P) and treated with $10 \mathrm{ng} / \mathrm{ml}$ TNF $\alpha$ or $50 \mathrm{ng} / \mathrm{ml} \mathrm{IFN} \gamma$ for 18 hours. (B) Histograms show mean \pm SEM relative to no treatment control cells. ${ }^{*} P<0.05$ vs. no tx; $n=3$. doi:10.1371/journal.pone.0031231.g002 knockdown is dependent on p53, markers of autophagy were assessed in $\mathrm{p}^{-/-}$HCT 116 cells transfected with siPHB. p53 $3^{-/-}$ cells showed increased LC3-II and GFP-LC3 puncta formation compared to WT cells (Figure 4A and 4C) as previously described [52]. $\mathrm{p} 53^{-1-}$ cells also exhibited increased beclin-1 protein expression compared to WT cells (Figure 4A and 4B). Knockdown of PHB caused a further increase in autophagy in $\mathrm{p} 53^{-/-}$cells, suggesting that siPHB-mediated autophagy is independent of p53 signaling. ER stress is not further increased in $\mathrm{p} 53^{-/-}$cells upon PHB knockdown (Figure S2).

PHB knockdown increases intracellular reactive oxygen species and induces mitochondrial depolarization

Autophagy is induced by multiple stimuli including damage to organelles such as the endoplasmic reticulum and mitochondria. Given that the predominant subcellular localization of PHB is in the mitochondria in most cell types studied to date, emerging data suggest that PHB plays a role in stabilizing components of the electron transport chain [38]. Depletion of PHB function has been shown to cause electron transport chain disruption and increased intracellular oxidative stress $[39,40]$. As an indicator of intracellular ROS, Caco2-BBE cells transfected with siPHB or siNeg ctl were loaded with the oxidation-sensitive dye $\mathrm{H}_{2} \mathrm{DCF}$ DA and assayed for DCF fluorescence 10, 20 and 30 minutes after loading using a plate reader. PHB knockdown caused a $30 \%$ increase in intracellular ROS compared to control cells at each time point measured (Figure 5A; No tx). Recent data has shown that specific gene mutations or deficiency in autophagy is linked to susceptibility to inflammatory bowel disease $[9,10,11,12]$. To mimic this situation, autophagy was inhibited using $100 \mathrm{nM}$ Baf A for 12 hours or RNAi against the autophagy gene ATG16L1. Efficiency of ATG16L1 knockdown is shown in Figure 5D. Inhibition of autophagy increased intracellular ROS in both control and siPHB-transfected cells; the magnitude, however, was significantly greater in siPHBtransfected cells at all time points (Figure 5A; Baf $\mathrm{A}$ and siATG16L). Caco2-BBE cells stably overexpressing PHB show reduced DCF fluorescence compared to vector-transfected cells (Figure 5B; No tx). In the setting of PHB overexpression coupled with autophagy inhibition (Baf A or siATG16L1), ROS levels are not further increased and in fact are decreased during loss of ATG16L1. These results suggest that PHB expression regulates intracellular ROS and that autophagy prevents the accumulation of ROS.

To determine whether the increase in intracellular ROS is associated with altered mitochondrial integrity, mitochondrial membrane potential (MMP) was assessed using JC-1 dye. As shown in Figure 5C, the percentage of Caco2-BBE cells testing positive for depolarized mitochondria increased from $\sim 29 \%$ of control cells to $\sim 41 \%$ of cells with PHB knockdown in the absence of inhibitors of autophagy (No tx, left panels), suggesting that loss of PHB negatively affects mitochondrial integrity. Inhibition of autophagy using Baf A or RNAi against ATG16Ll increased the percentage of control cells (Figure 5C, top panels) with mitochondrial membrane depolarization from $\sim 29 \%$ to $\sim 36 \%$ (Baf A) and $\sim 51 \%$ (siATG16L1). In cells with silenced PHB, treatment with Baf $\mathrm{A}$ increased the percentage of cells with mitochondrial membrane depolarization from $\sim 41 \%$ to $\sim 51 \%$ and siATG16L1 increased the percentage to $\sim 56 \%$ (Figure $5 \mathrm{C}$, bottom panels). These results suggest that loss of PHB function induces mitochondrial dysfunction and that inhibition of autophagy increases the number of cells with altered mitochondrial function within the cell population. 
A

Caco2-BBE

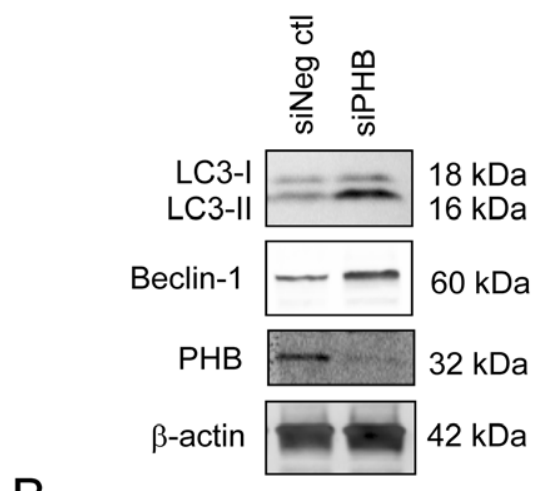

B

LC3-II/LC3-I
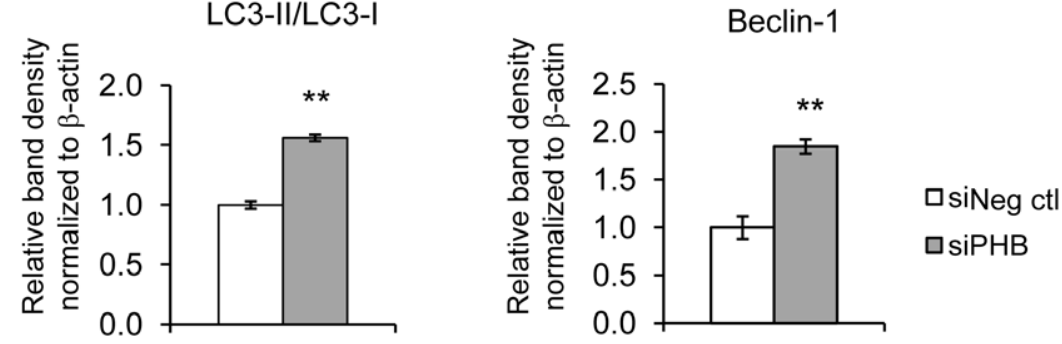

C
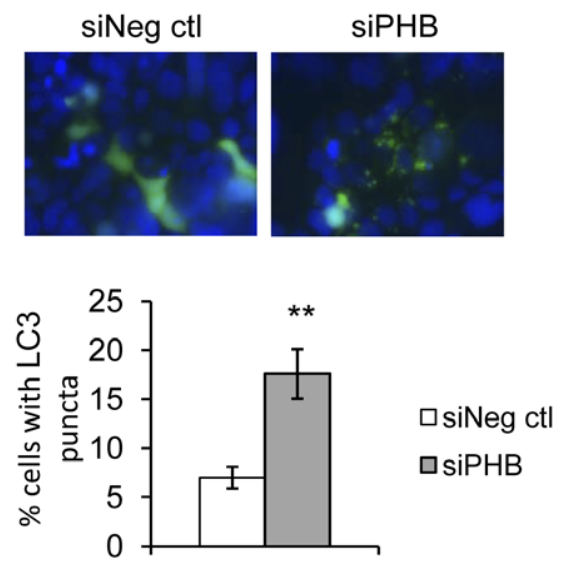

Figure 3. Knockdown of PHB induces autophagy. Markers of autophagy were assessed in Caco2-BBE cells transfected with either siNeg ctl or siPHB for 96 hours. (A) Representative Western blots showing LC3I/LC3II, Beclin-1 or $\beta$-actin (loading control) expression. PHB protein levels were assessed to determine efficiency of knockdown. (B) Histograms show mean \pm SEM. ${ }^{* *} P<0.01$ vs. siNeg ctl; $n=3$ (C) Fluorescent micrographs of cells expressing pSelect-GFP-LC3 (green) and stained with DAPI (blue) to visualize nuclei. Quantification of cells with punctuate GFP-LC3. Histograms show mean \pm SEM. ${ }^{* *} P<0.01$ vs. siNeg $\mathrm{ctl}, \mathrm{n} \geq 5$ fields with $>50$ cells/field. doi:10.1371/journal.pone.0031231.g003

\section{Silencing of PHB expression reduces cell viability}

Cell cytoxicity was assessed by measuring the release of lactate dehydrogenase. Across all treatments, Caco2-BBE cells transfected with siPHB were less viable than control cells (Figure 6A). Inhibition of autophagy using Baf A further decreased viability in siPHB-transfected cells compared to control cells. RNAi against the autophagy gene ATG16L1 reduced viability in both siPHB and siNeg ctl cells, but the effect was greater in siPHB cells. These results suggest that $\mathrm{PHB}$ knockdown reduces cell viability and that autophagy acts to promote cell survival. The effect of
PHB knockdown to reduce cell viability was exacerbated by the pro-inflammatory cytokines TNF $\alpha$ and IFN $\gamma$ (Figure 6A). IFN $\gamma$ reduced viability in cells with $\mathrm{PHB}$ knockdown versus control cells, but in combination with autophagy inhibition IFN $\gamma$ had no further effect than inhibition of autophagy alone. In contrast, $\mathrm{TNF} \alpha$ dramatically reduced cell viability in siPHB-transfected cells with inhibited autophagy (Figure 6A), supporting the concept that the IFN $\gamma$ autophagy pathway is distinct from that of $\mathrm{TNF} \alpha$. These results suggest that autophagy is important for cell survival when $\mathrm{TNF} \alpha$ levels are high and when $\mathrm{PHB}$ 
A HCT116

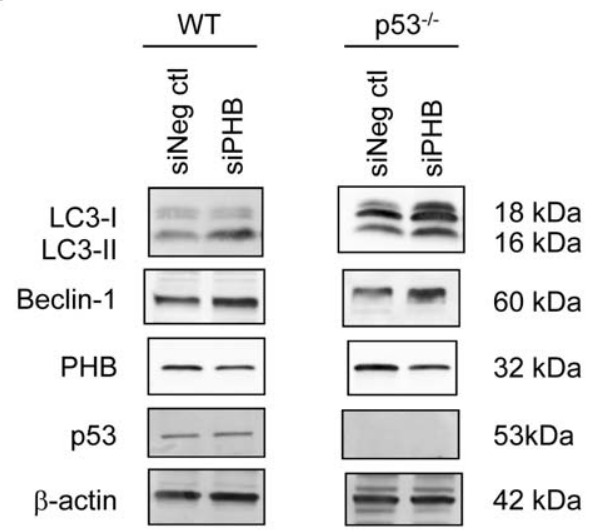

B

LC3-II/LC3-I

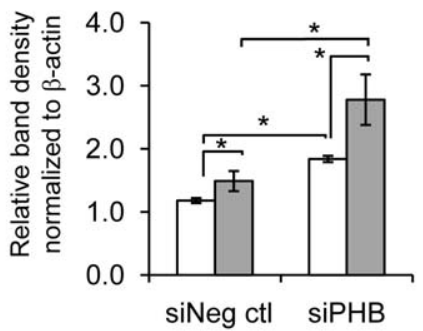

C

WT

p5 $53^{-1-}$
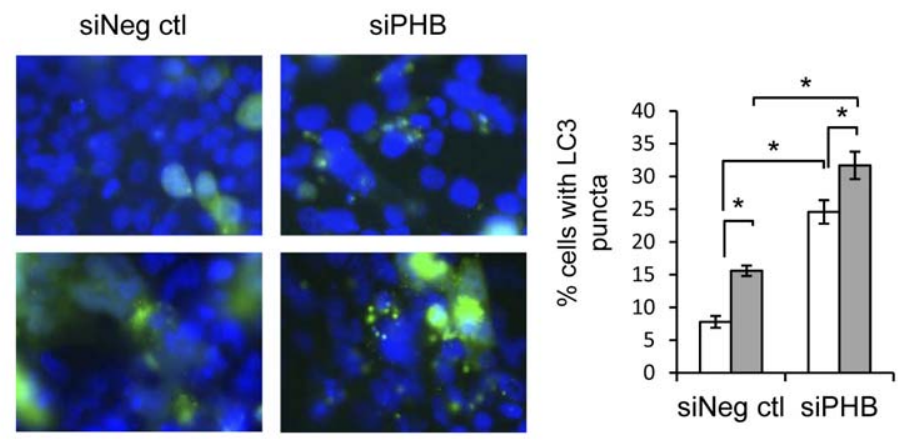

Beclin-1

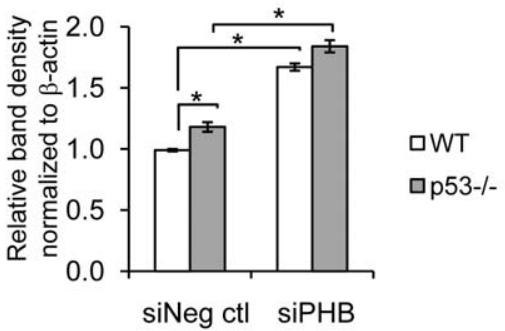

口WT

口p53-/-

Figure 4. Knockdown of PHB using siRNA induces autophagy independently of p53 in HCT116 cells. Markers of autophagy were assessed in HCT116 cells transfected with either siNeg ctl or siPHB for 96 hours. (A) Representative Western blot showing LC3I/LC3II , Beclin-1 or $\beta$ actin (loading control) expression. PHB protein levels were assessed to determine efficiency of knockdown. (B) Histograms show mean \pm SEM relative to no treatment control cells. ${ }^{*} P<0.05$ vs. siNeg $\mathrm{ctl} ; \mathrm{n}=3$. (C) Fluorescent micrographs of cells expressing pSelect-GFP-LC3 (green) and stained with DAPI (blue) to visualize nuclei. Quantification of cells with punctuate GFP-LC3. Histograms show mean \pm SEM. ${ }^{*} P<0.05$ vs. siNeg ctl, $\mathrm{n}=5$ fields with $>50$ cells/field.

doi:10.1371/journal.pone.0031231.g004

expression is reduced, a scenario present during intestinal inflammation.

To determine whether cell death induced by PHB knockdown involves apoptosis, cleaved Caspase-3 protein expression and TUNEL staining were determined. Levels of cleaved Caspase-3 protein were increased during $\mathrm{PHB}$ knockdown and further increased by autophagy inhibition and $\mathrm{TNF} \alpha$ treatment (Figure 6B). The percent TUNEL positive cells was increased during PHB knockdown and further increased by autophagy inhibition and TNF $\alpha$ treatment (Figure 6C), suggesting that cell death is at least partially due to apoptosis. To determine whether increased intracellular ROS is associated with increased cell death during autophagy inhibition and $\mathrm{TNF} \alpha$ treatment, DCF fluorescence was measured in cells transfected with or without siPHB and treated with $\mathrm{TNF} \alpha$ and autophagy inhibitors. Figure 6D shows that knockdown of PHB increased ROS levels for all treatment groups compared to control. TNF $\alpha$ treatment during inhibition of autophagy causes a further increase in intracellular ROS, which is further exaggerated in cells with PHB knockdown.

Treatment with NAC, a ROS scavenger, prevents siPHBinduced mitochondrial stress-related autophagy

To determine whether mitochondria are indeed recycled during siPHB-induced autophagy, siPHB and GFP-LC3 co-transfected 
A

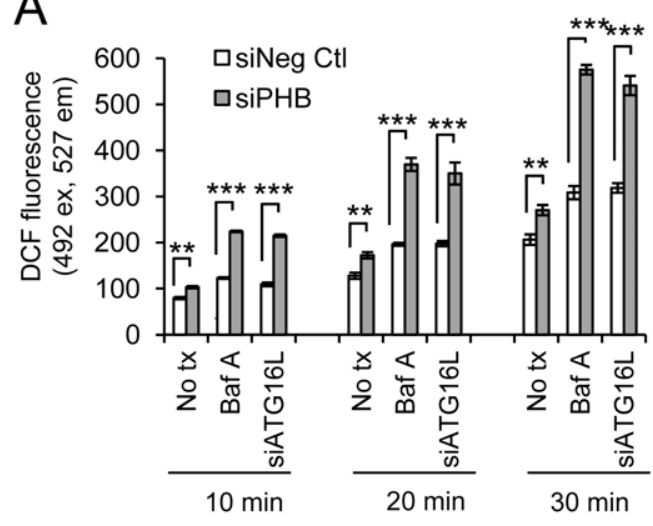

B

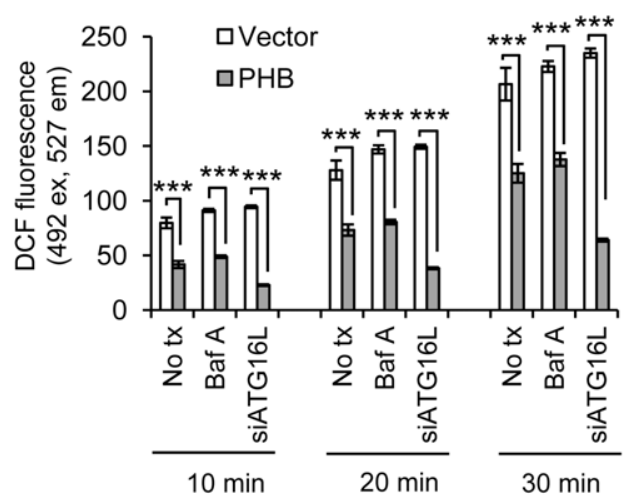

C
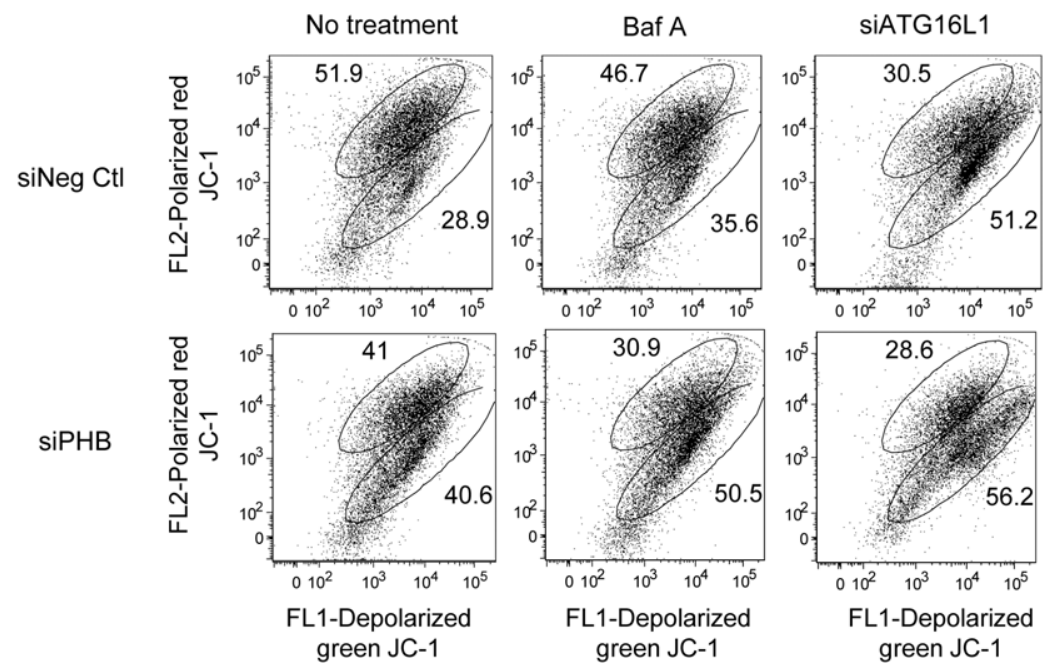

D

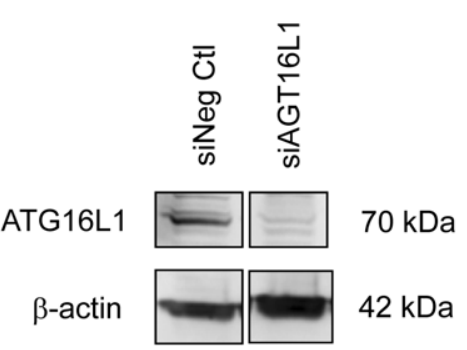

Figure 5. PHB knockdown increases intracellular reactive oxygen species and induces mitochondrial depolarization. (A) DCF fluorescence was measured in Caco2-BBE cells transfected with siRNA for 96 hours and treated as indicated for 18 hours. Cells were loaded with DCFH-DA for 10 minutes and fluorescence was determined 10,20 and 30 minutes after loading using a plate reader to assess intracellular ROS levels. ${ }^{* *} P<0.01$, ${ }^{* * *} P<0.001 ; n=16$ from two separate experiments. (B) DCF fluorescence was measured in Caco2-BBE cells stably transfected with $p E G F P N 1$ expression vector or pEGFPN1-PHB as in (A). ${ }^{* * *} P<0.001 ; n=8$. (C) Mitochondrial membrane depolarization was measured in Caco2-BBE cells stained with JC-1 dye as demonstrated by a shift from red (intact MMP) to green (depolarized) fluorescence detected by flow cytometry. The data shown are a representative result from one of three experiments. (D) ATG16L1 protein levels were assessed by Westen blotting to determine efficiency of knockdown.

doi:10.1371/journal.pone.0031231.g005

cells were labeled with MitoTracker, a mitochondria-specific dye (red fluorescence). Laser scanning confocal microscopy revealed significant co-localization of mitochondria with GFP autophagosomes in cells with silenced PHB compared to control cells (Figure 7A, upper panels versus middle panels), suggesting mitophagy. To determine whether increased intracellular ROS is the mechanism by which PHB knockdown induces autophagy, cells were treated with $1.0 \mathrm{mM}$ NAC for 24 hours prior to collection. The addition of NAC prevented the increase in GFPLC3 puncta, indicative of autophagosomes, by PHB knockdown and prevented the co-localization of GFP-LC3 with mitochondria (Figure 7A, lower panels).

Caco2-BBE cells were transfected with siPHB for 96 hours and treated with $1.0 \mathrm{mM}$ or $10.0 \mathrm{mM}$ NAC for 24 hours starting at 72 hours post-transfection. PHB knockdown stimulated the conversion of LC3I to LC3II compared to siNeg ctl cells 
A

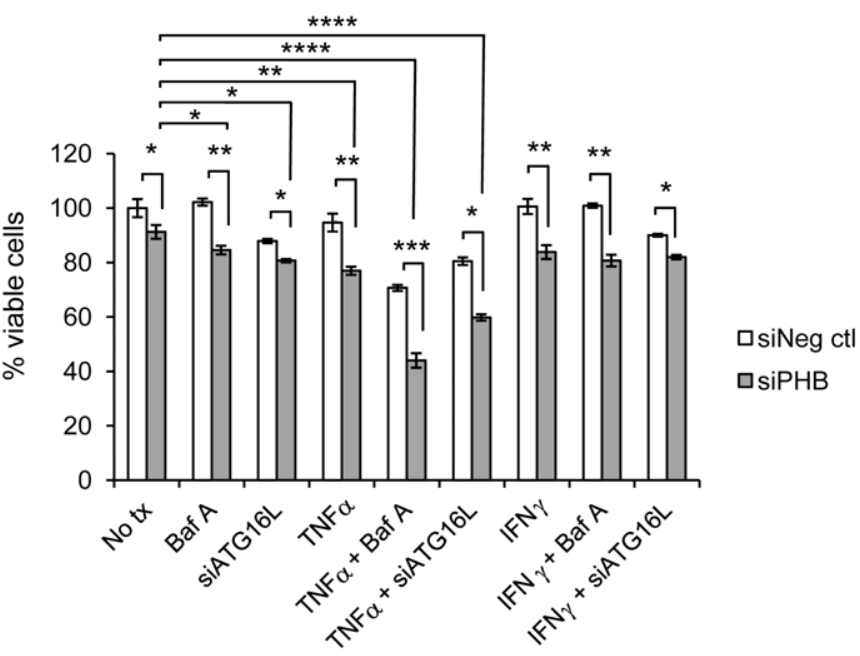

B
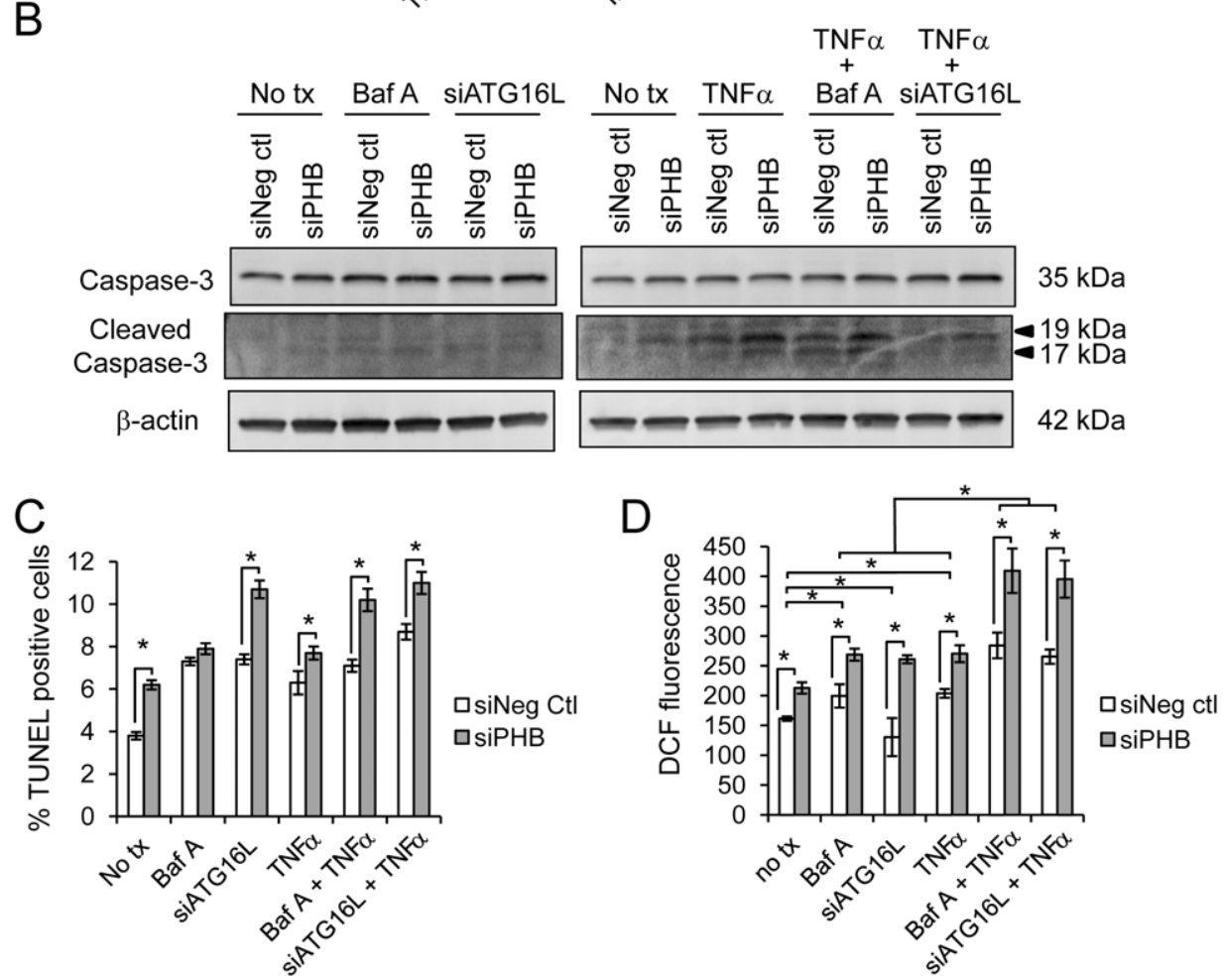

Figure 6. Silencing of PHB expression reduces cell viability. (A) Cytotoxicity as measured by LDH test of Caco2-BBE cells transfected with siRNA for 96 hours and treated as indicated for 18 hours. ${ }^{*} P<0.05,{ }^{* *} P<0.01,{ }^{* * *} P<0.001 ;{ }^{* * * *} P<0.0001$ vs. all other treatments; $\mathrm{n}=3$ experiments run in quadruplicate. (B) Representative Western blot showing cleaved Caspase-3 or $\beta$-actin (loading control) expression. (C) Percent TUNEL-positive Caco2-BBE cells. A minimum of 5 fields were counted for each treatment. ${ }^{*} P<0.05$. (D) DCF fluorescence was measured in Caco2-BBE cells transfected with siRNA for 96 hours and treated as indicated for 18 hours. Cells were loaded with DCFH-DA for 10 minutes and fluorescence was determined 10 minutes after loading. ${ }^{*} P<0.05 ; n=8$.

doi:10.1371/journal.pone.0031231.g006

(Figure 7B), indicating autophagy, as shown in Figure 3A. The addition of NAG prevented siPHB-induced conversion of LC3I to LC3II in a dose-dependent manner (Figure 7B). These results suggest that silencing of PHB expression increases intracellular oxidative stress, which subsequently induces mitophagy. DCF fluorescence was measured to ensure that NAC treatment reduced intracellar ROS (Figure 7G).

\section{Discussion}

In addition to the emerging role of autophagy in controlling bacterial invasion $[15,53,54]$, autophagy recycling represents the final tier of mitochondrial quality control. Multiple studies have reported mitochondrial dysfunction in Crohn's disease and ulcerative colitis $[16,17,18,19]$ and mouse models of colitis [20,21]. Mitochondria are important regulators of autophagy and apoptosis. Exogenous ROS and cytokines such as TNF $\alpha$, both of which are increased during IBD, promote cellular injury and autophagy via mitochondrial ROS generation [29,30,31]. We show here that PHB modulates autophagy in intestinal and colonic epithelial cells. Reduced PHB protein expression by RNA interference induces autophagy of mitochondria and treatment with NAC, a ROS scavenger, prevents siPHB-induced autophagy. These results suggest that loss of PHB expression induces 
A

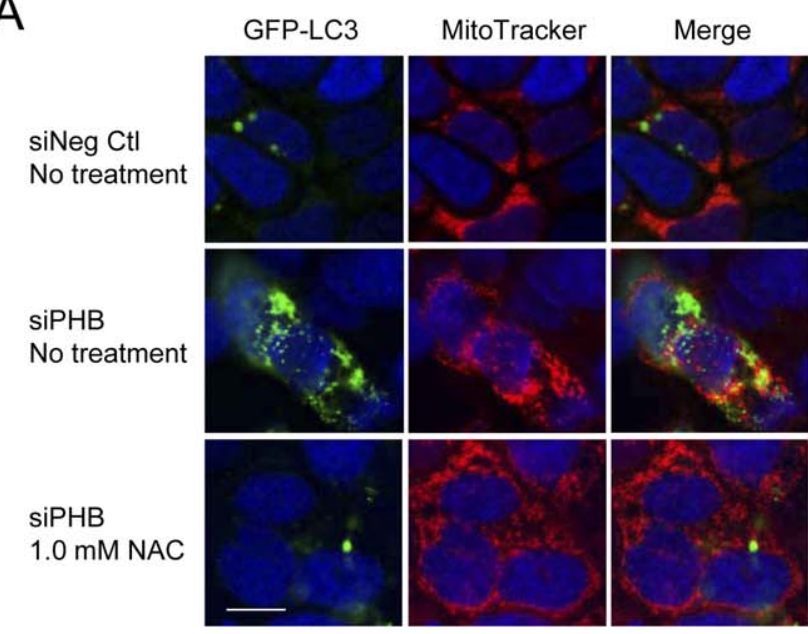

B

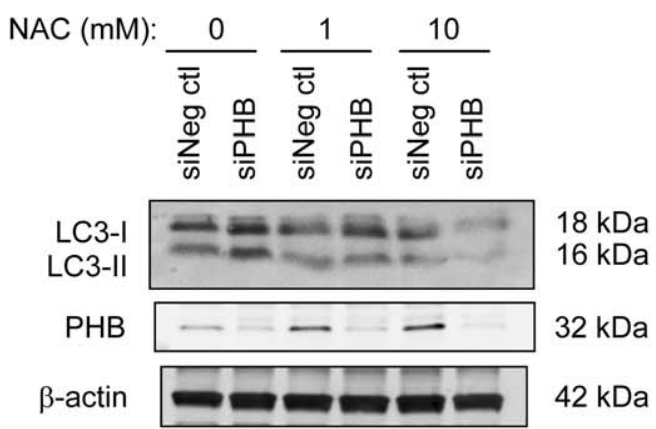

C

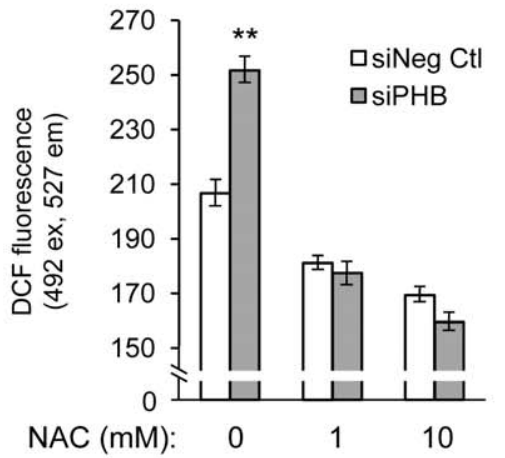

Figure 7. Treatment with NAC, a ROS scavenger, prevents siPHB-induced mitochondrial stress-related autophagy. (A) Caco2-BBE cells were co-transfected with siPHB or siNeg Ctl and GFPLC3 (green fluorescence) and incubated with MitoTracker dye (red fluorescence, mitochondria). Cells were incubated with $1.0 \mathrm{mM}$ NAC for 24 hours prior to collection. Merged confocal images demonstrate mitophagy (yellow fluorescence). Cells were stained with DAPI to visualize nuclei (blue). Bar $=10 \mu \mathrm{M}$. (B) Representative Western blot showing LC3I/LC3II or $\beta$-actin (loading control) expression. The data shown are a representative result from one of three experiments. (C) DCF fluorescence was measured in Caco2-BBE cells transfected with siPHB for 96 hours and treated with NAC for 18 hours. Cells were loaded with DCFH-DA for 10 minutes and fluorescence was determined 20 minutes after loading using a plate reader to assess intracellular ROS levels. ${ }^{* *} P<0.01, \mathrm{n}=8$.

doi:10.1371/journal.pone.0031231.g007

autophagy via increased intracellular ROS. PHB knockdown induces mitochondrial membrane depolarization, suggesting mitochondrial damage, and increased intracellular ROS which is likely generated via dysfunctional respiration, all of which are exacerbated by inhibition of autophagy. Therefore, autophagy plays a protective role during conditions when $\mathrm{PHB}$ expression is low in intestinal epithelial cells.

We have previously shown that expression of PHB protein is reduced in mucosa during active inflammatory bowel disease and in Caco2-BBE cells treated with $\mathrm{TNF} \alpha$ or exogenous oxidants $[37,42]$. It has been demonstrated that $\mathrm{TNF} \alpha$ and IFN $\gamma$ induce autophagy in intestinal epithelium $[31,55]$ and that autophagy can attenuate inflammatory responses, thereby maintaining intestinal homeostasis [56]. PHB protein levels inversely correlated with $\mathrm{TNF} \alpha$ and IFN $\gamma$-induced autophagy in Caco2-BBE and HCT116 cells. Furthermore, exogenous PHB expression reduced basal autophagy and $\mathrm{TNF} \alpha$-induced autophagy, suggesting that expression of PHB modulates the cellular autophagic response. One potential mechanism whereby $\mathrm{PHB}$ regulates beclin-1, the first mammalian gene shown to induce autophagy, is through the transcription factor E2F1. The beclin-1 promoter contains a putative E2F1 binding site [57] and PHB has been shown to regulate E2F1 activity [35]. Future studies will investigate this possibility. It is compelling to speculate that reduced $\mathrm{PHB}$ expression coupled with dysfunctional autophagy, an emerging susceptibility trait in Crohn's disease [13,14,15], could render epithelial cells unable to recycle damaged mitochondria and thus they succumb to cell death. It has been shown that when less than $66 \%$ of the mitochondria within a cell are damaged, autophagy predominates to restore cell homeostasis, while apoptosis is triggered when the percentage increases to involve most of the mitochondrial population $[58,59]$. The likely reason cell viability is decreased during PHB knockdown coupled with autophagy inhibition or $\mathrm{TNF} \alpha$ treatment is that the threshold of damaged mitochondria exceeds the capacity of the cell.

Our finding that decreased PHB levels coupled with inhibited autophagy increased cell death has important clinical implications. Patients with inflammatory bowel disease exhibit decreased expression of PHB in intestinal epithelial cells [37,41]. Dysfuntion in autophagy genes such as ATG16L1, IRGM, and LRRK2, are emerging as potential susceptibility traits in patients with inflammatory bowel disease $[9,10,11,12]$. We speculate that decreased expression of PHB during active inflammatory bowel disease is a signal to the epithelial cell that there is inflammatory stress and that autophagy is subsequently induced to maintain cell viability and return homeostasis. Thus, these findings support an important role of autophagy in intestinal health and lend further insight into the mechanisms of dysfunctional autophagy via $\mathrm{PHB}$ in inflammatory bowel diseases. In the setting of PHB overexpression coupled with autophagy inhibition, ROS levels were not further increased and in fact are decreased during loss of ATG16L1. These data support the therapeutic concept of repletion of PHB levels in the setting of dysfunctional autophagy.

Basal autophagy was reduced by PHB overexpression in Caco2BBE cells. We have shown previously that exogenous PHB expression in Caco2-BBE cells induced the expression of glutathione-S-transferase $\pi$, an antioxidant enzyme that catalyzes the conjugation of electrophiles to GSH [37], and modulates the activity of nuclear factor erythroid 2-related factor 2 (Nrf2), a transcriptional regulator of antioxidant response [42]. $\mathrm{PHB}$ overexpression may reduce basal autophagy through an increased antioxidant response on ROS production during normal physiological respiration. This issue warrants further investigation.

Although PHB protein levels inversely correlated with IFN $\gamma$ induced autophagy, overexpression of PHB in Caco2-BBE cells did not affect IFN $\gamma$-induced autophagy. This is in contrast to the response to $\mathrm{TNF} \alpha$. $\mathrm{NF}-\kappa \mathrm{B}$, a major downstream signaling 
pathway of $\mathrm{TNF} \alpha$, has been implicated in modulating autophagy [60]. Our previous work demonstrated that exogenous PHB expression reduced basal and $\mathrm{TNF} \alpha$-stimulated $\mathrm{NF}-\kappa \mathrm{B}$ activation [42]. It is possible that this effect on NF- $\kappa B$ reduces $T N F \alpha-$ induced autophagy as compared to IFN $\gamma$ since IFN $\gamma$ is not a major activator of $\mathrm{NF}-\kappa \mathrm{B}$. Furthermore, $\mathrm{TNF} \alpha$ promotes autophagy via mitochondrial ROS generation [31]. TNF $\alpha$ treatment during inhibition of autophagy exacerbated intracellular ROS levels in cells with knockdown of PHB. Since PHB overexpression did not decrease IFN $\gamma$ induced autophagy, this would suggest that the IFN $\gamma$ autophagy pathway is distinct from that of TNF $\alpha$. Exogenous PHB expression can likely prevent $\mathrm{TNF} \alpha$-induced autophagy since $\mathrm{PHB}$ overexpression reduces ROS generation. The mechanism whereby PHB overexpression dampens basal and $\mathrm{TNF} \alpha$-induced, but not IFN $\gamma$-induced, autophagy requires further investigation.

It is widely accepted that the tumor suppressor p53 regulates autophagy depending upon is subcellular localization [49]. Normal levels of p53 maintain a tonic inhibition of autophagy; autophagy is induced via a reduction in cytoplasmic p53 levels [61]. p53 can activate genes that induce autophagy including damage-regulated autophagy modulator (DRAM) and sestrins 1 and 2 [62]. Since Caco2-BBE cells have mutated p53, we included WT and p53 null HCT116 colonic epithelial cells to assess the involvement of p53 in autophagy mediated by PHB knockdown. PHB levels inversely correlated with $\mathrm{TNF} \alpha$ - or IFN $\gamma$-induced autophagy regardless of p53 status. As previously described [52], p53 $3^{-1-}$ HCT 116 cells exhibited increased autophagy compared to WT cells. p53 knockout initially induces autophagy by ER stress followed by mitophagy [52]. Knockdown of PHB caused a further increase in autophagy in $\mathrm{p} 53^{-/-}$cells, suggesting that autophagy mediated by PHB knockdown is independent of p53. ER stress markers indicate that ER stress was not further increased upon PHB knockdown. We speculate that p53 null cells with PHB knockdown likely show more autophagy due to a combination of ER stress-induced autophagy (due to p53 knockout) and mitophagy (due to loss of PHB expression and p53 knockout).

In conclusion, we demonstrate that the mitochondrial protein PHB modulates autophagy in intestinal epithelial cells via intracellular ROS signaling. Decreased PHB expression coupled with inhibition of autophagy, renders intestinal epithelial cells unable to maintain cell homeostasis and susceptible to mitochondrial damage and cytotoxicity. These findings have elucidated a molecular pathway whereby increased ROS by decreased PHB may enhance inflammation in patients with inflammatory bowel disease.

\section{Materials and Methods}

\section{Cell culture}

The Caco2-BBE human intestinal epithelial cell line was used as in vitro model of polarized intestinal epithelium. The Caco2-BBE cell line was obtained from the American Type Culture Collection (ATCG; Manassas, VA). Since Caco2-BBE cells have mutated p53, wild-type (WT) and p53 null [51] HCT116 human colonic epithelial cells were utilized to assess the involvement of p53 in autophagy induction by PHB knockdown. Cells were grown in Dulbecco's modified Eagle's medium (DMEM; Caco2-BBE) or Modified McCoy's media (HCT116) supplemented with penicillin (40 mg/l), streptomycin (90 mg/l), and 10\% fetal bovine serum. Caco2-BBE cells were plated on permeable supports to allow the cells to polarize (pore size, $0.4 \mu \mathrm{m}$; transwell-clear polyester membranes; Costas life science, Acton, MA), while HCT116 cells were plated on plastic. All experiments performed on Caco2-BBE cells were between passages 32 and 45 .

\section{PHB knockdown and overexpression}

Cells were transiently transfected with Stealth $\mathrm{RNAi}^{\mathrm{TM}}$ against PHB 1 (5'-CAGAAUGUCAACAUGACAGUGGGCA - $3^{\prime}$ ) or Stealth RNAi ${ }^{\text {TM }}$ siRNA Negative Control Med GC (Invitrogen, Carlsbad, CA) at $20 \mu \mathrm{m}$ concentration. Caco2-BBEs were transfected using Amaxa ${ }^{\circledR}$ Cell Line Optimization Nucleofector ${ }^{\circledR}$ Kit T (Lonza, Basel, Switzerland), while HCT 116 were transfected with LipofectAmine 2000 (Invitrogen). Cells were transfected with siRNA for 96 hours (Figure S1A-D). For PHB overexpression studies, Caco2-BBE cells were transiently transfected with either pEGFPN1 expression vector $(\mathrm{V})$ or pEGFPN1-PHB (P) for 72 hours (generation of the pEGFPN1-PHB construct is described below).

\section{Autophagy activation and inhibition}

Serum deprived cells were treated with $10 \mathrm{ng} / \mathrm{ml}$ recombinant human TNF $\alpha$ (R\&D Systems, Minneapolis, MN) or $50 \mathrm{ng} / \mathrm{ml}$ recombinant human IFN $\gamma$ (eBioscience, San Diego, CA) for 18 hours. When treating Caco2-BBE cells, TNF $\alpha$ was administered to the basolateral chamber, while IFN $\gamma$ was administered to the apical and basolateral chambers. To inhibit autophagy, cells were treated with $100 \mathrm{nM}$ Bafilomycin $\mathrm{A}_{1}$ (Baf A; Sigma-Aldrich, St. Louis, MO) $24 \mathrm{~h}$ prior to collection or co-transfected with $20 \mu \mathrm{m}$ siATG16L1 (5'-AUUACUGCGAGAUAGGGAACGCUUG-3'). Efficiency of siRNA knockdown was assessed by Western blotting (Figure 5D). Cells were treated with 1.0 or $10.0 \mathrm{mM} \mathcal{N}$-acetyl- $L$-cysteine (Sigma-Aldrich), a ROS scavenger, for 24 hours prior to collection.

\section{Generation of stably-transfected Caco2-BBE cell expressing pEGFPN1-PHB}

A single PHB PCR product corresponding to the entire coding region of PHB (818 bp) was generated from Caco2-BBE cells using an antisense primer with a mutated PHB stop codon, underlined (5'-AATTGGATCGCGTCGCTGGGGCAGCTGGA-3'). The PCR product was ligated into pEGFPN1 vector (Clontech, Palo Alto, CA) using the Quick Ligation Kit (New England Biolabs, Ipswich, MA) and sequenced. Caco2-BBE cells were transfected with pEGFPN1-PHB or empty pEGFPN1 vector using Lipofectamine 2000 (Invitrogen) and the transfected clones were selected under $0.12 \%$ geneticin (Sigma-Aldrich), and fluorescent cells were isolated using flow cytometry (fluorescence-activated cell sorting).

\section{Western blot analysis}

Total protein was collected from Caco2-BBE or HCT116 cells in lysis buffer containing $1 \%$ Triton X-100, 1\% Nonidet P-40 (vol/vol), $1 \mathrm{mM}$ EDTA, $1 \mathrm{mM}$ sodium orthovanadate, $1 \mathrm{mM}$ sodium fluoride, and $1 \mu \mathrm{l} / \mathrm{ml}$ mammalian protease inhibitor cocktail (BioExpress, Kaysville, UT) to obtain total protein extracts. The samples were separated by sodium dodecyl sulfatepolyacrylamide gel electrophoresis (SDS-PAGE) using laemmli's $2 \times$ SDS sample buffer and AnyKD ${ }^{\text {TM }}$ gradient polyacrylamide gels (Bio-Rad, Hercules, CA) followed by electrotransfer to nitrocellulose membranes (Bio-Rad). Membranes were incubated with primary antibodies at $4{ }^{\circ} \mathrm{C}$ overnight and subsequently incubated with corresponding peroxidase-conjugated secondary antibodies. Membranes were washed and immunoreactive proteins were detected using Amersham ECL Plus ${ }^{\text {TM }}$ reagent (GE Healthcare, Piscataway, NJ). Blots were reprobed with anti- $\beta$-actin (Sigma-Aldrich) antibody. Mouse monoclonal PHB antibody was obtained from Thermo Fisher (Fremont, CA), mouse monoclonal GFP, mouse monoclonal ClpP, and rabbit polyclonal PKR antibodies from Santa Cruz Biotechnology (Santa Cruz, CA), 
rabbit polyclonal LC3 and ATG16L1 antibodies from SigmaAldrich, rabbit polyclonal Beclin-1, cleaved Caspase- 3, p53, Cpn60, and all ER stress antibodies from Cell Signaling Technology (Danvers, MA).

\section{Fluorescence microscopy}

pSelect-NGFP-LC3 was purchased from InvivoGen (San Diego, CA). pSelect-GFP-LC3 was co-transfected into Caco2-BBE and HCT 116 cells with either siNeg Ctl or siPHB for 96 hours. Cells were fixed in buffered $4 \%$ paraformaldehyde for 20 minutes, washed with phosphate buffered saline, and counterstained with DAPI to visualize nuclei. Stained cells were mounted in phenylenediamine/glycerol $(1: 1)$ and analyzed by fluorescence microscopy (Zeiss Axioskope Plus).

\section{Confocal microscopy}

Caco2-BBE cells grown to confluency on filters were transfected with pSelect-NGFP-LC3 for 96 hours and incubated with $100 \mathrm{nM}$ MitoTracker dye (Invitrogen) for 15 minutes. A subset of cells was treated with $1.0 \mathrm{mM} \mathrm{NAC}$ for 24 hours prior to collection. Cells were fixed in buffered $4 \%$ paraformaldehyde for 20 minutes, washed with PBS, and counterstained with DAPI to visualize nuclei. Samples were mounted in $p$-phenylenediamine/glycerol $(1: 1)$ and analyzed by confocal microscopy.

\section{DCF assay}

As a measure of intracellular ROS, conversion of the nonionic, nonpolar 2', 7' -dichlorodihydrofluorescein diacetate $\left(\mathrm{H}_{2} \mathrm{DCFDA}\right.$ : Invitrogen) to fluorescent $2^{\prime}, 7^{\prime}$-dichlorofluorescein (DCF) was measured. Caco2-BBE cells were loaded with $10 \mu \mathrm{M} \mathrm{H} \mathrm{H}_{2}$ DCFDA for 10 minutes and fluorescence was quantitated 10, 20 and 30 minutes later according to the manufacturer's protocol using a plate reader.

\section{Cytotoxicity test}

Lactate dehydrogenase (LDH) cytotoxicity detection kit (Clontech, Mountain View, CA) was used to measure cell viability. LDH determines the secretion of $\mathrm{LDH}$ into the culture medium from dead or membrane-damaged cells. Caco2-BBE cells transfected with siRNA for 96 hours and were treated with $\mathrm{TNF} \alpha, \mathrm{IFN} \gamma$, or Baf A alone or in combination for 18 hours prior to collection. An aliquot of $100 \mu \mathrm{l}$ of culture media was added to $100 \mu \mathrm{l}$ of $\mathrm{LDH}$ reagent and \% cytotoxicity and \% viable cells were measured according to the manufacturer's protocol using a plate reader.

\section{Terminal deoxynucleotidyl transferase-mediated} deoxyuridine triphosphate nick-end labeling (TUNEL) staining

TUNEL staining was performed on confluent Caco2-BBE cells as described by the manufacturer's protocol (Roche, Indianapolis, IN).

FACS analysis of mitochondrial membrane potential Mitoprobe $^{\text {TM }}$ JC-1 assay kit was used (Molecular Probes, Eugene, $\mathrm{OR}$ ) to detect mitochondrial membrane depolarization.

\section{References}

1. Dalby KN, Tekedereli I, Lopez-Berestein G, Ozpolat B (2010) Targeting the prodeath and prosurvival functions of autophagy as novel therapeutic strategies in cancer. Autophagy 6: 322-329.

2. Gurusamy N, Das DK (2009) Autophagy, redox signaling, and ventricular remodeling. Antioxid Redox Signal 11: 1975-1988.

3. Mizushima N, Levine B, Cuervo AM, Klionsky DJ (2008) Autophagy fights disease through cellular self-digestion. Nature 451: 1069-1075.
JC-1 is a cationic dye which gives orange emission upon aggregates in normal polarized mitochondria and in monomeric form gives green fluorescence in the depolarized mitochondria. Briefly, $4 \times 10^{5}$ Caco2-BBE cells transfected with siNeg Ctl or siPHB for 96 hours were trypsinized, washed and resuspended in a final volume of $1 \mathrm{ml}$ of warm media. Cells were stained with $2 \mu \mathrm{m}$ JC-1 dye for 15 minutes at $37^{\circ} \mathrm{C}$ protected from light. After incubation, cells were washed and resuspended in $500 \mu \mathrm{l}$ of phosphate buffered saline and were analyzed on Becton Dickinson FACS Canto II flow cytometer.

\section{Statistical analysis}

Values are expressed as mean \pm SEM. Comparisons between $\mathrm{TNF} \alpha$ or IFN $\gamma$ treatment or siPHB versus control were analyzed by unpaired Student's $t$-test. Comparisons between PHB RNAi and autophagy inhibition were analyzed by two-way analysis of variance to test for a significant interaction between PHB knockdown and impaired autophagy. Subsequent pair wise comparisons used Bonferroni post-hoc tests to test for significant differences between two particular groups. $\mathrm{p}<0.05$ was considered statistically significant in all analyses.

\section{Supporting Information}

Figure S1 PHB knockdown does not induce a mitochondrial unfolded protein response in Caco2-BBE cells. Representative Western blot showing expression of the mitochondrial stress proteins Cpn60, PKR, ClpP. PHB protein levels were assessed to determine efficiency of knockdown.

(TIF)

Figure S2 ER stress markers are not further increased in p53-null HGT116 cells upon PHB knockdown. Representative Western blots showing expression of various ER stess markers. Deletion of p53 causes an increase in ER stress as previously reported [52].

(TIF)

\section{Acknowledgments}

The authors thank the late Dr. Shanthi V. Sitaraman from Emory University, Atlanta, GA for her dedication, generosity, and inspiration with this project. We are grateful to Dr. Ajay Goel from Baylor Research Institute, Baylor University Medical Center, Dallas, TX for providing to us the HCT116 cell line. We thank Dr. Shanthi Srinivasan from Emory University, Atlanta, GA for helpful scientific discussions. The authors thank Sandra Clayton for her assistance with confocal imaging through the Baylor Institute for Immunological Research Imaging Core, Baylor Research Institute, Dallas, TX.

\section{Author Contributions}

Conceived and designed the experiments: ASK RFS ALT. Performed the experiments: ASK LDB ALT. Analyzed the data: ASK LDB ALT. Contributed reagents/materials/analysis tools: CRB ALT. Wrote the paper: ASK LAF RFS ALT.

4. Beau I, Mehrpour M, Codogno P (2011) Autophagosomes and human diseases. Int J Biochem Cell Biol 43: 460-464.

5. Glick D, Barth S, Macleod KF (2010) Autophagy: cellular and molecular mechanisms. J Pathol 221: 3-12.

6. Mehrpour M, Esclatine A, Beau I, Codogno P (2010) Autophagy in health and disease. 1. Regulation and significance of autophagy: an overview. Am J Physiol Cell Physiol 298: C776-785. 
7. Sartor RB, Muehlbauer M (2007) Microbial host interactions in IBD: implications for pathogenesis and therapy. Curr Gastroenterol Rep 9: 497-507.

8. Roda G, Sartini A, Zambon E, Calafiore A, Marocchi M, et al. (2010) Intestinal epithelial cells in inflammatory bowel diseases. World J Gastroenterol 16: $4264-4271$.

9. The Wellcome Trust Case Control Consortium (2007) Genome-wide association study of 14,000 cases of seven common diseases and 3,000 shared controls. Nature 447: 661-678.

10. Rioux JD, Xavier RJ, Taylor KD, Silverberg MS, Goyette P, et al. (2007) Genome-wide association study identifies new susceptibility loci for Crohn disease and implicates autophagy in disease pathogenesis. Nat Genet 39: 596-604.

11. Umeno J, Asano K, Matsushita T, Matsumoto T, Kiyohara Y, et al. (2011) Meta-analysis of published studies identified eight additional common susceptibility loci for Crohn's disease and ulcerative colitis. Inflamm Bowel Dis. Epub ahead of print.

12. Barrett JC, Hansoul S, Nicolae DL, Cho JH, Duerr RH, et al. (2008) Genomewide association defines more than 30 distinct susceptibility loci for Crohn's disease. Nat Genet 40: 955-962.

13. Kuballa P, Huett A, Rioux JD, Daly MJ, Xavier RJ (2008) Impaired autophagy of an intracellular pathogen induced by a Crohn's disease associated ATG16L1 variant. PLoS One 3: e3391.

14. Saitoh T, Fujita N, Jang MH, Uematsu S, Yang BG, et al. (2008) Loss of the autophagy protein Atg16L1 enhances endotoxin-induced IL-1beta production. Nature 456: 264-268.

15. Cadwell K, Liu JY, Brown SL, Miyoshi H, Loh J, et al. (2008) A key role for autophagy and the autophagy gene Atg1611 in mouse and human intestinal Paneth cells. Nature 456: 259-263.

16. O'Morain C, Smethurst P, Levi J, Peters TJ (1985) Subcellular fractionation of rectal biopsy homogenates from patients with inflammatory bowel disease. Scand J Gastroenterol 20: 209-214.

17. Vanderborght M, Nassogne MC, Hermans D, Moniotte S, Seneca S, et al. (2004) Intractable ulcerative colitis of infancy in a child with mitochondrial respiratory chain disorder. J Pediatr Gastroenterol Nutr 38: 355-357.

18. Restivo NL, Srivastava MD, Schafer IA, Hoppel CL (2004) Mitochondrial dysfunction in a patient with crohn disease: possible role in pathogenesis. J Pediatr Gastroenterol Nutr 38: 534-538.

19. Sifroni KG, Damiani GR, Stoffel C, Cardoso MR, Ferreira GK, et al. (2010) Mitochondrial respiratory chain in the colonic mucosal of patients with ulcerative colitis. Mol Cell Biochem 342: 111-115.

20. Damiani CR, Benetton CA, Stoffel C, Bardini KC, Cardoso VH, et al. (2007) Oxidative stress and metabolism in animal model of colitis induced by dextran sulfate sodium. J Gastroenterol Hepatol 22: 1846-1851.

21. Tirosh O, Levy E, Reifen R (2007) High selenium diet protects against TNBSinduced acute inflammation, mitochondrial dysfunction, and secondary necrosis in rat colon. Nutrition 23: 878-886.

22. Du G, Mouithys-Mickalad A, Sluse FE (1998) Generation of superoxide anion by mitochondria and impairment of their functions during anoxia and reoxygenation in vitro. Free Radic Biol Med 25: 1066-1074

23. Lih-Brody L, Powell SR, Collier KP, Reddy GM, Cerchia R, et al. (1996) Increased oxidative stress and decreased antioxidant defenses in mucosa of inflammatory bowel disease. Dig Dis Sci 41: 2078-2086.

24. McKenzie SJ, Baker MS, Buffinton GD, Doe WF (1996) Evidence of oxidantinduced injury to epithelial cells during inflammatory bowel disease. J Clin Invest 98: 136-141.

25. Rachmilewitz D, Stamler JS, Bachwich D, Karmeli F, Ackerman Z, et al. (1995) Enhanced colonic nitric oxide generation and nitric oxide synthase activity in ulcerative colitis and Crohn's disease. Gut 36: 718-723.

26. Shiratora Y, Aoki S, Takada H, Kiriyama H, Ohto K, et al. (1989) Oxygenderived free radical generating capacity of polymorphonuclear cells in patients with ulcerative colitis. Digestion 44: 163-171.

27. Chen Y, Gibson SB (2008) Is mitochondrial generation of reactive oxygen species a trigger for autophagy? Autophagy 4: 246-248.

28. Scherz-Shouval R, Elazar Z (2007) ROS, mitochondria and the regulation of autophagy. Trends Cell Biol 17: 422-427.

29. Scherz-Shouval R, Elazar Z (2011) Regulation of autophagy by ROS: physiology and pathology. Trends Biochem Sci 36: 30-38.

30. Djavaheri-Mergny M, Amelotti M, Mathieu J, Besancon F, Bauvy C, et al. (2006) NF-kappaB activation represses tumor necrosis factor-alpha-induced autophagy. J Biol Chem 281: 30373-30382.

31. Baregamian N, Song J, Bailey CE, Papaconstantinou J, Evers BM, et al. (2009) Tumor necrosis factor-alpha and apoptosis signal-regulating kinase 1 control reactive oxygen species release, mitochondrial autophagy, and c-Jun $\mathrm{N}$-terminal kinase/p38 phosphorylation during necrotizing enterocolitis. Oxid Med Cell Longev 2: 297-306.

32. Mizushima N, Klionsky DJ (2007) Protein turnover via autophagy: implications for metabolism. Annu Rev Nutr 27: 19-40.

33. Jupe ER, Liu XT, Kiehlbauch JL, McClungJK, Dell'Orco RT (1995) Prohibitin antiproliferative activity and lack of heterozygosity in immortalized cell lines. Exp Cell Res 218: 577-580.
34. Wang S, Fusaro G, Padmanabhan J, Chellappan SP (2002) Prohibitin colocalizes with $\mathrm{Rb}$ in the nucleus and recruits $\mathrm{N}-\mathrm{CoR}$ and HDACl for transcriptional repression. Oncogene 21: 8388-8396.

35. Wang S, Zhang B, Faller DV (2002) Prohibitin requires Brg-1 and Brm for the repression of E2F and cell growth. Embo J 21: 3019-3028.

36. Rastogi S, Joshi B, Fusaro G, Chellappan S (2006) Camptothecin induces nuclear export of prohibitin preferentially in transformed cells through a CRM1-dependent mechanism. J Biol Chem 281: 2951-2959.

37. Theiss AL, Idell RD, Srinivasan S, Klapproth JM, Jones DP, et al. (2007) Prohibitin protects against oxidative stress in intestinal epithelial cells. Faseb J 21: 197-206.

38. Artal-Sanz M, Tavernarakis N (2009) Prohibitin and mitochondrial biology. Trends Endocrinol Metab 20: 394-401.

39. Schleicher M, Shepherd BR, Suarez Y, Fernandez-Hernando C, Yu J, et al (2008) Prohibitin-1 maintains the angiogenic capacity of endothelial cells by regulating mitochondrial function and senescence. J Cell Biol 180: 101-112.

40. Tsutsumi T, Matsuda M, Aizaki H, Moriya K, Mivoshi H, et al. (2009) Proteomics analysis of mitochondrial proteins reveals overexpression of a mitochondrial protein chaperon, prohibitin, in cells expressing hepatitis $\mathrm{C}$ virus core protein. Hepatology 50: 378-386.

41. Hsieh SY, Shih TC, Yeh CY, Lin CJ, Chou YY, et al. (2006) Comparative proteomic studies on the pathogenesis of human ulcerative colitis. Proteomics 6 : 5322-5331.

42. Theiss AL, Jenkins AK, Okoro NI, Klapproth JM, Merlin D, et al. (2009) Prohibitin inhibits tumor necrosis factor alpha-induced nuclear factor-kappa B nuclear translocation via the novel mechanism of decreasing importin alpha3 expression. Mol Biol Cell 20: 4412-4423.

43. Theiss AL, Laroui H, Obertone TS, Chowdhury I, Thompson WE, et al. (2011) Nanoparticle-based therapeutic delivery of prohibitin to the colonic epithelial cells ameliorates acute murine colitis. Inflamm Bowel Dis 17: 1163-1176.

44. Theiss AL, Vijay-Kumar M, Obertone TS, Jones DP, Hansen JM, et al. (2009) Prohibitin is a novel regulator of antioxidant response that attenuates colonic inflammation in mice. Gastroenterology 137: 199-208.

45. Henschke P, Vorum H, Honore B, Rice GE (2006) Protein profiling the effects of in vitro hyperoxic exposure on fetal rabbit lung. Proteomics 6: 1957-1962.

46. Liu X, Ren Z, Zhan R, Wang X, Wang X, et al. (2009) Prohibitin protects against oxidative stress-induced cell injury in cultured neonatal cardiomyocyte. Cell Stress Chaperones 14: 311-319.

47. Kondo Y, Kanzawa T, Sawaya R, Kondo S (2005) The role of autophagy in cancer development and response to therapy. Nat Rev Cancer 5: 726-734.

48. Klionsky DJ, Abeliovich H, Agostinis P, Agrawal DK, Aliev G, et al. (2008) Guidelines for the use and interpretation of assays for monitoring autophagy in higher eukaryotes. Autophagy 4: 151-175.

49. Maiuri MC, Galluzzi L, Morselli E, Kepp O, Malik SA, et al. (2010) Autophagy regulation by p53. Curr Opin Cell Biol 22: 181-185.

50. Lin-Lee YC, Tatebe S, Savaraj N, Ishikawa T, Tien Kuo M (2001) Differential sensitivities of the MRP gene family and gamma-glutamylcysteine synthetase to prooxidants in human colorectal carcinoma cell lines with different p53 status. Biochem Pharmacol 61: 555-563.

51. Bunz F, Hwang PM, Torrance C, Waldman T, Zhang Y, et al. (1999) Disruption of p53 in human cancer cells alters the responses to therapeutic agents. J Clin Invest 104: 263-269.

52. Tasdemir E, Maiuri MC, Galluzzi L, Vitale I, Djavaheri-Mergny M, et al. (2008) Regulation of autophagy by cytoplasmic p53. Nat Cell Biol 10: 676-687.

53. Homer CR, Richmond AL, Rebert NA, Achkar JP, McDonald C (2010) ATG16L1 and NOD2 interact in an autophagy-dependent antibacterial pathway implicated in Crohn's disease pathogenesis. Gastroenterology 139: 1630-1641.

54. Lapaquette P, Darfeuille-Michaud A (2010) Abnormalities in the handling of intracellular bacteria in Crohn's disease. J Clin Gastroenterol 44 Suppl 1: S26-29.

55. Chang YP, Chen CL, Chen SO, Lin YS, Tsai CC, et al. (2011) Autophagy facilitates an IFN-gamma response and signal transduction. Microbes Infect 13: 888-894.

56. Fujishima Y, Nishiumi S, Masuda A, Inoue J, Nguyen NM, et al. (2011) Autophagy in the intestinal epithelium reduces endotoxin-induced inflammatory responses by inhibiting NF-kappaB activation. Arch Biochem Biophys 506: 223-235.

57. Tang H, Da L, Mao Y, Li Y, Xu Z, et al. (2009) Hepatitis B virus X protein sensitizes cells to starvation-induced autophagy via up-regulation of beclin 1 expression. Hepatology 49: 60-71.

58. Alva AS, Gultekin SH, Baehrecke EH (2004) Autophagy in human tumors: cell survival or death? Cell Death Differ 11: 1046-1048.

59. Sasnauskiene A, Kadziauskas J, Vezelyte N, Jonusiene V, Kirveliene V (2009) Apoptosis, autophagy and cell cycle arrest following photodamage to mitochondrial interior. Apoptosis 14: 276-286.

60. Xiao G (2007) Autophagy and NF-kappaB: fight for fate. Cytokine Growth Factor Rev 18: 233-243.

61. Galluzzi L, Morselli E, Kepp O, Vitale I, Pinti M, et al. (2011) Mitochondrial Liaisons of p53. Antioxid Redox Signal.

62. Green DR, Kroemer G (2009) Cytoplasmic functions of the tumour suppressor p53. Nature 458: 1127-1130. 